\title{
Scaling for the SOL/separatrix $\chi_{\perp}$ following from the Heuristic Drift Model for the power scrape-off layer width
}

\author{
A. Huber ${ }^{1}$ and A. V. Chankin ${ }^{2}$ \\ ${ }^{I}$ Forschungszentrum Jülich GmbH, Institut für Energie- und Klimaforschung - Plasmaphysik, \\ Partner of the Trilateral Euregio Cluster (TEC), D-52425 Jülich, Germany \\ ${ }^{2}$ Max-Planck-Institut für Plasmaphysik, D-85748 Garching, Germany
}

\begin{abstract}
A simple two-point representation of the tokamak scrape-off layer (SOL) in the conduction limited regime, based on the parallel and perpendicular energy balance equations in combination with the heat flux width predicted by a heuristic drift based model, was used to derive a scaling for the cross-field thermal diffusivity $\chi_{\perp}$. For fixed plasma shape and neglecting weak power dependence indexes $1 / 8$, the scaling $\chi_{\perp} \propto P_{S O L} /\left(n B_{\theta} R^{2}\right)$ is derived.
\end{abstract}

\section{Introduction}

Understanding transport in fusion experiments, through both electron and ion channels, is essential for planning and predicting future devices. In this contribution we concentrate on the electron heat channel in tokamaks which is larger than the neoclassical prediction by about two orders of magnitude. It is attributed to anomalous cross-field transport caused by microturbulence. Recently, intensive measurements of the power decay length in SOL, $\lambda_{q}$, were carried out on JET and ASDEX Upgrade [1] with the use of infrared thermography. Based on these data, an empirical scaling for $\lambda_{q}$ was derived. A comparison of this scaling with a heuristic particle drift-based model [2] (HD model) shows satisfactory agreement in both absolute magnitude and power dependencies. The HD model assumes that the SOL width $\left(\Delta_{\mathrm{SOL}}\right)$ is determined by ion $\nabla B$ drift as well as that $\Delta_{S O L}=\lambda_{q}$ leading to the scaling $\lambda_{q} \sim 2\left(\frac{a}{R}\right) \rho_{p}$, where $\rho_{p}$ is the ion poloidal gyro radius. It is assumed in this model however that the anomalous perpendicular electron thermal diffusivity is the dominant source of heat flux across the separatrix.

Perpendicular anomalous transport can be characterised by the cross-field thermal diffusivity $\chi_{\perp}$. In this work the scaling for $\chi_{\perp}$ is derived from the heat flux width used in the HD model. We use similar assumptions as in the HD model that the dominant heat transport across the separatrix is due to anomalous electron thermal diffusion. If the ion channel is important, then it is implicitly assumed that it obeys similar laws as the electron channel. 


\section{Power balance in the SOL}

In the simple SOL model one assumes equality between the (mostly conductive) power flow across the separatrix and parallel heat flow to the target, assuming in addition an exponential decay of the latter. The power crossing the separatrix, $P_{S O L}$, is given by:

$$
q_{\perp} S_{\perp} \approx-n \chi_{\perp} \nabla_{\perp} T S_{\perp}
$$

where $S_{\perp}$ is the surface area of the LCFS which defines the surface over which power and particle fluxes from the core region cross into the SOL:

$$
S_{\perp}=4 \pi^{2} R a \sqrt{\frac{1+\kappa^{2}}{2}}
$$

with $\kappa=b / a$ plasma elongation, $a$ the horizontal minor radius and $b$ the vertical minor radius of an elliptical plasma in the poloidal plane.

The power flowing to the divertor targets:

$$
q_{||} S_{\|}=K_{||} \nabla_{\|} T S_{||} \approx-n \chi_{\|} \nabla_{\|} T S_{||}
$$

where $S_{\|}$is the SOL cross-sectional area perpendicular to $\vec{B}$ for the heat flux, given by:

$$
S_{||} \approx 2 \pi R \lambda_{q} \sin \gamma \approx 2 \pi R \lambda_{q} B_{\theta} / B
$$

where $\gamma$ is the field line angle at the outboard mid-plane and $B_{\theta}$ and $B$ are the poloidal and total magnetic field strengths.

In the equations above $\chi_{\|}$and $\chi_{\perp}$ are parallel and perpendicular thermal diffusivities, $q_{\|}$and $q_{\perp}$ the heat fluxes, $K_{\|}=n \chi_{\|}$and $K_{\perp}=n \chi_{\perp}$ are parallel and perpendicular thermal conductivities, $n$ is electron density and $T=T_{e}$ is electron temperature.

The balance between the perpendicular (from the plasma core into the SOL) and parallel (along the SOL flux tube to the divertor target) flow of power $\left(P_{S O L}=S_{\perp} q_{\perp}=S_{\|} q_{\|}\right)$can be described by the power balance equations:

$$
\begin{aligned}
& q_{\|}=n \chi_{\|} \nabla_{\|} T=\frac{B}{B_{\theta}} \frac{P_{S O L}}{4 \pi R \lambda_{q}}, \\
& q_{\perp}=n \chi_{\perp} \frac{T}{\lambda_{T}}=\frac{P_{S O L}}{4 \pi^{2} R a\left(\left(1+\kappa^{2}\right) / 2\right)^{1 / 2}} .
\end{aligned}
$$


Here the approximation $\nabla_{\perp} T \approx-T / \lambda_{T}$ was used, where $\lambda_{T}$ is the electron perpendicular temperature decay length in the SOL. Additionally it is assumed that the heat power crossing the SOL goes to both inner and outer divertor targets.

In the conduction-limited regime the plasma heat flux parallel to the magnetic field can be written as $[3,4]$ :

$$
q_{\|}=\frac{P_{S O L}}{S_{\|}}=-k_{0 e} T_{e}^{5 / 2} \nabla_{\|} T_{e}
$$

where the electron thermal conductivity constant, $\mathrm{k}_{0 \mathrm{e}}$, has a value of $\sim 2046$ (for ions $\mathrm{k}_{0 \mathrm{i}} \sim 59$ ) (see Appendix) with $T_{e}$ in $\mathrm{eV}$. The electron heat conduction is assumed to be the dominant channel for power flux to the target. The upstream separatrix temperature, $T_{e, u}=T_{e, s e p}$, may be evaluated using a two point model of the SOL transport [5]:

$$
T_{e, u}{ }^{7 / 2} \approx T_{e, t^{\frac{7}{2}}}+\left(\frac{7}{2}\right) P_{S O L} L_{\|} /\left(S_{\|} k_{0, e}\right)
$$

where $T_{e, u}$ and $T_{e, t}$ are the upstream and downstream (target) values of $T_{e}, L_{\|}$is the upstream to target connection length. $T_{e, t}$ will be neglected below, assuming high recycling divertor conditions where $T_{e}$ falls strongly towards the target. It should be mentioned here that for very low density, high power, low collisionality plasmas the dependence $q_{\|} \propto T_{e}{ }^{7 / 2}$ doesn't apply (see Section 5) .

From Eq. (6) one obtains the upstream electron temperature at the separatrix, $T_{e, s e p}=T_{e, u}$ :

$$
T_{e, s e p}=\left(\frac{\left(\frac{7}{2}\right) P_{S O L} L_{\|}}{4 \pi R \lambda_{q}{ }^{B} \theta /{ }_{B} k_{0, e}}\right)^{2 / 7}, \text { with } T_{e} \text { in } \mathrm{eV}
$$

as well as for the heat flux crossing the separatrix:

$$
P_{S O L}=\frac{4 \pi R \lambda_{q} k_{0, e} T_{e, s e p}{ }^{7 / 2}}{\left(\frac{7}{2}\right) L_{\|}} \frac{B_{\theta}}{B} .
$$

In Eq. (7) the factor $4 \pi R \lambda_{q}$ in the denominator reflects an assumption that the heat power crossing the SOL goes to both inner and outer divertor.

Within the framework of a two-point model the relation between the mid-plane electron temperature decay length $\left(\lambda_{T e}=\lambda_{T}\right)$ and target heat flux decay length $\left(\lambda_{\mathrm{q}}\right)$ is given by $\lambda_{T e}=$ 
$7 / 2 \lambda_{q}$. Combining equations (4) and (7) to eliminate $T_{\text {sep }}$ and taking $L_{\|}=(\pi / 2) \quad q R$ [2], corresponding to the case of poloidally constant flux density across separatrix, we arrive at:

$n \chi_{\perp}=\frac{0.144}{e} k_{0, e} e^{2 / 7} \frac{P_{S O L}^{5 / 7} \lambda_{q}^{9 / 7}}{q_{c y l} l^{4 / 7} R^{9 / 7} a^{5 / 7}}\left(1+\kappa^{2}\right)^{-5 / 14}$

i.e. $\chi_{\perp}$ increases with $\lambda_{q}$ and $P_{S O L}$ and decreases with $n_{s e p}, q_{c y l}$ and $R$.

\section{Heat Transport using Goldston's power SOL width}

The HD model has the following power flux decay length $\lambda_{\mathrm{q}}$ at the outer mid-plane:

$\lambda_{q}=5671 \times P_{S O L} 1 / 8 \frac{\left(1+\kappa^{2}\right)^{5 / 8} a^{17 / 8} B^{1 / 4}}{I_{p}{ }^{9 / 8} R}\left(\frac{2 \bar{A}}{1+\bar{Z}}\right)^{7 / 16}\left(\frac{Z_{e f f}+4}{5}\right)^{1 / 8}$,

where dimensional variables are expressed in S.I. units, $Z_{\text {eff }}$ and $\bar{Z}$ are the "effective charge" and "average charge" of all ions. In the case of only single ion species $\bar{Z}=Z_{\text {eff }}$. The equation (10) can also be written as:

$\lambda_{q}=0.00036 \times P_{S O L}{ }^{1 / 8} q_{c y l}{ }^{9 / 8}(R / a)^{1 / 8} B^{-7 / 8} \frac{1}{\left(1+\kappa^{2}\right)^{1 / 2}}\left(\frac{2 \bar{A}}{1+\bar{Z}}\right)^{7 / 16}\left(\frac{Z_{e f f}+4}{5}\right)^{1 / 8}$

where $q_{c y l}=\left(2 \pi a^{2} B\right) /\left(R I_{p} \mu_{0}\right)\left(1+\kappa^{2}\right) / 2$ is the cylindrical approximation for the safety factor. Substituting this $\lambda_{q}$ into equation (9) one obtains for the perpendicular thermal conduction:

$K_{\perp}=n \chi_{\perp}=3.36 \times 10^{13} k_{0, e^{2 / 7}} \frac{P_{S O L}{ }^{7 / 8} q_{c y l}{ }^{7 / 8}}{\left(1+\kappa^{2}\right) R^{9 / 8} a^{7 / 8} B^{9 / 8}}\left(\frac{2 \bar{A}}{1+\bar{Z}}\right)^{9 / 16}\left(\frac{Z_{e f f}+4}{5}\right)^{9 / 56}$.

For parameters typical of the SOL in JET: $R=3 \mathrm{~m}, a=0.94 \mathrm{~m}, Z_{\text {eff }}=1.2-1.5, B=2.7 \mathrm{~T}, q_{c y}=3.0$, and $\kappa=1.68$, with $P_{S O L}=10 \mathrm{MW}$ and $n_{\text {sep }}=2.5 \times 10^{19} \mathrm{~m}^{-3}$, the evaluated perpendicular thermal diffusivity for the deuterium plasma $(\bar{A}=2)$ gives $\chi_{\perp} \approx 1.56 \mathrm{~m}^{2} / \mathrm{s}$, which is typically used in edge $2 \mathrm{D}$ codes for modelling of the SOL and divertor.

Substituting the electron thermal conductivity constant (see appendix) into equation (12), one obtains: 


$$
K_{\perp}=n \chi_{\perp}=2.97 \times 10^{14} \frac{P_{S O L}{ }^{7 / 8} q_{c y l} l^{7 / 8}}{\left(1+\kappa^{2}\right) R^{9 / 8} a^{7 / 8} B^{9 / 8}}\left(\frac{2 \bar{A}}{1+\bar{Z}}\right)^{9 / 16} f\left(Z_{e f f}\right)^{1 / 8},
$$

where $f\left(Z_{\text {eff }}\right)$ is either $f^{H D}\left(Z_{\text {eff }}\right)$ or $f^{a p p}\left(Z_{\text {eff }}\right)$ (see Appendix), and we replaced $Z_{i}$ with $Z_{\text {eff }}$ in these expressions.

\section{Dependence of the perpendicular heat transport on main plasma parameters}

Neglecting weak power dependences with index $1 / 8$ as well as a weak dependence of $Z_{\text {eff }}$, the electron thermal conductivity as well as the thermal diffusivity could be given by the following approximation:

$$
K_{\perp} \propto \frac{P_{S O L}}{B_{\theta} R^{2}} \times \sqrt{\frac{\bar{A}}{1+\bar{Z}}}, \quad \quad \chi_{\perp} \propto \frac{P_{S O L}}{n B_{\theta} R^{2}} \times \sqrt{\frac{\bar{A}}{1+\bar{Z}}} .
$$

This result doesn't depend on the density profile in the SOL. In particular, an assumption of the density width being determined by the ion $\nabla B$ drift in the SOL, used in HD model, doesn't influence the result. The width of the power footprint at the target is determined by radial upstream $T_{e}$ decay length in the SOL, which, in turn, is determined by anomalous electron thermal conduction given by equations $(13,14)$.

\section{Applicability of the derived scaling for $\chi_{\perp}$}

In this section we discuss the limits of applicability of the derived scaling for $\chi_{\perp}$, as well as the limits of some assumptions of the HD model. The notion of the 'SOL width', or 'heat flux width', used in the HD model, being equal to the distance in the SOL beyond which no significant power flux to the target can be observed, is reduced to the 'particle width': the characteristic distance over which ions can propagate into the SOL driven by the $\nabla B$ drift. In its extreme form, it can be illustrated by a cartoon in Fig.1. It relates to the situation where charged particles only exist within the layer $\Delta_{S O L}$, whereas the electron temperature decay length $\lambda_{T}$ is much greater that $\Delta_{S O L}$. Despite the near constant profile of $T_{e}^{7 / 2}$ which describes the conductive power deposition within the framework of the two points model, the width of the power deposition onto the target is given by $\Delta_{S O L}$ and not the $2 / 7 \lambda_{T}$ as would follow from the two point model for a conduction limited regime. This is because, due to zero 
plasma density, the plasma is no longer collisional beyond $\Delta_{S O L}$ and the power flux starts to scale with $n T_{e}^{3 / 2}$, hence it is also zero.

In the experiment, one usually operates with (exponential) power decay lengths, often corrected by introducing a Gaussian profile to take into account the perpendicular thermal diffusion or the leakage into the private-flux-region (PFR) [1]. This is the more realistic situation compared to the idealistic situation we considered above. The 'SOL width' situation then corresponds to the case of the density decay length $\lambda_{n}$ much shorter than $2 / 7 \lambda_{T}$, as shown in Fig. 2a. This, however, is only the necessary condition to relate power decay length $\lambda_{q}$ with $\lambda_{n}$. If at distance $\lambda_{n}$ from the separatrix the plasma remains collisional, so the power flux law $T_{e}^{7 / 2}$ still applies, $\lambda_{n}$ becomes irrelevant and the heat flux $\Delta_{S O L}$ must be above $\lambda_{n}$, up to the distance at which density falls so low that power flux starts scaling with , and owing to negligible density the power flux is also negligible.

An alternative situation is shown by the cartoon in Fig.2b. Here $2 / 7 \lambda_{T}<<\lambda_{n}$ and $\Delta_{S O L}=2 / 7 \lambda_{T}$. The decay lengths of electron density and temperature at the torus midplane were investigated on JET and Alcator C-Mod with the help of the reciprocating probe [6]. It was found that the ratio of the upstream decay lengths, $\lambda_{n} / \lambda_{T}$, varied between 0.5 and 1.0 supporting the view that the case shown in Fig.2b is the most realistic one. However, recent near SOL measurements in ASDEX Upgrade with the high precision edge Thomson scattering system revealed even larger $\lambda_{n}$ compared to $\lambda_{T}$ in a wide range of experimental conditions, with $\lambda_{n} / \lambda_{T}$ around $\approx 1.4[7]$.

Hence, out of the two situations we considered in Figs. 2a,b, only in one of them one can relate power flux width with $\lambda_{n}$ : the case with $\lambda_{n}<2 / 7 \lambda_{T}$ plus the condition that the plasma density at distance $\lambda_{n}$ from the separatrix is so low that the power flux scales with $n T_{e}^{3 / 2}$ rather than with $T_{e}^{7 / 2}$. We consider such a situation as unrealistic, and therefore assume that $\lambda_{q}$ should be determined by $\lambda_{T}$ rather than by $\lambda_{n}$. We would also like to point out, however, that some experiments [6,7] reveal a close proportionality between $\lambda_{T}$ and $\lambda_{n}$ in the near SOL. Since $\lambda_{T}$ is determined by anomalous electron heat transport (which is also one of the assumptions of the model [2]), it is quite possible that cross-field particle and heat transport are determined by the same, or similar, turbulent transport mechanism. 
In any case, the discussion whether $\lambda_{q}$ is more driven by $\lambda_{n}$ or $\lambda_{T}$ is indeed a hot topic at the moment in the fusion community and further investigations are required.

We should also mention here that the heuristic particle drift-based model shows an excellent agreement with the experimentally derived empirical scaling for $\lambda_{q}[1]$ in both absolute magnitude and power dependences.

\section{Summary}

The $\lambda_{\mathrm{q}}$, used in a heuristic drift (HD) model was adopted to derive an expression for perpendicular electron thermal conductivity assuming that it is the upstream electron temperature profile, rather than density profile, that determines the power decay length (or power width) on the target. For a deuterium plasma a realistic value of $\chi_{\perp} \approx 1.9 \mathrm{~m}^{2} / \mathrm{s}$, often used in the edge $2 \mathrm{D}$ codes, is obtained. The derived $\chi_{\perp}$ approximately scales with $P_{S O L}$, and is inversely proportional to poloidal magnetic field $B_{\theta}, n_{\text {sep }}$ and $R^{2}: \chi_{\perp} \propto P_{S O L} /\left(n B_{\theta} R^{2}\right)$, with the neglect of weak power dependencies (1/8), plasma shape parameters and a weak dependence on $\bar{Z}$.

\section{Acknowledgements}

This work has been carried out within the framework of the EUROfusion Consortium and has received funding from the Euratom research and training programme 2014-2018 under grant agreement No 633053. The views and opinions expressed herein do not necessarily reflect those of the European Commission. One of the authors, A. V. Chankin, appreciates discussions with Dr. J. Brodrick on equations for parallel electron heat transport.

\section{Appendix}

In this section we review expressions for parallel electron heat conductivity coefficient $k_{o, e}$.

The collisional electron heat flux parallel to the magnetic field for the case of singly charged ions $\left(Z_{i}=1\right)$ is given by [4]:

$$
q_{\|}=-3.16 \frac{n_{e} T_{e} \tau_{e}}{m_{e}} \nabla_{\|} T_{e},
$$

where the electron collision time is given by [4]:

$$
\tau_{e}=\frac{3 \sqrt{m_{e}} T_{e}^{3 / 2}}{4 \sqrt{2 \pi} \ln \Lambda e^{4} n_{e}},
$$


where $\ln \Lambda$ is the Coulomb logarithm.

From (15) and (16) the electron heat flux can be expressed in term of the electron heat conductivity coefficient

$q_{\|}=-k_{0} e^{T} e^{\frac{5}{2}} \nabla_{\|} T_{e}$

Numerically, electron thermal conductivity constant can be expressed by

$k_{0, e}=\frac{30692}{Z_{i} \ln \Lambda}=2046\left[\frac{W}{m e V^{\frac{7}{2}}}\right]$

In Eq. (17) $T_{e}$ should be given in $\mathrm{eV}$. The numerical values above are given for the Coulomb $\operatorname{logarithm} \ln \Lambda=15$. Similar, but a more approximate expression can be found in [5] (Eq. (4.83)).

For ion species with $Z_{i} \neq 1$, the electron thermal conductivity constant should be corrected [4]:

$k_{0, e}=2046 \frac{\frac{\gamma_{0}}{3.16}}{Z_{i}}=2046 \cdot f\left(Z_{i}\right)\left[\frac{W}{m e V^{\frac{7}{2}}}\right]$,

where $\gamma_{0}=3.1616,4.89 ; 6.064 ; 6.92,12.5$ for $Z_{i}=1,2 ; 3 ; 4$ and $\infty$, respectively [4]. Epperlein and Haines [8] have found inaccuracies in Braginski's analysis, affecting primarily the heat conductivity coefficient for $Z_{i}=\infty$, which has to be 13.58 instead of 12.5 .

Taking into account the corrected $\gamma_{0}$, we interpolate the function $f\left(Z_{i}\right)=\frac{\gamma_{0} / 3.16}{Z_{i}}$ by $f^{\text {app }}\left(Z_{i}\right)=$ $\frac{4.295}{Z_{i}} \times\left(\frac{Z_{i}+0.21}{Z_{i}+4.2}\right)$ which diverges from Braginski's coefficients for $Z_{i} \leq 4$ no more than by $1.2 \%$ and gives the correct ("Eppelein and Haines") value for $Z_{i}=\infty$. A similar expression $f^{H D}\left(Z_{i}\right)=\left(\frac{5}{Z_{i}+4}\right)$ was used in [1], which is larger by factors of 1.001, 1.089, 1.119, 1.134 and 1.164 than $f^{\text {app }}$ for $Z_{i}=1,2,3,4$ and $\infty$, respectively.

The electron thermal conductivity constant as well as the electron heat flux parallel to the magnetic field then becomes:

$k_{0, e}=2046 \cdot \frac{4.295}{Z_{i}} \times\left(\frac{Z_{i}+0.21}{Z_{i}+4.2}\right)\left[\frac{W}{m e V^{\frac{7}{2}}}\right]$,

$q_{\|}=\frac{8.788 \times 10^{3}}{Z_{i}} \times\left(\frac{Z_{i}+0.21}{Z_{i}+4.2}\right) T_{e}{ }^{5 / 2} \nabla_{\|} T_{e}\left[\frac{W}{m^{2}}\right]$.

Note that $k_{o, e}$. is determined by both e-i and e-e collisions. There is no general expression for these coefficients for a multi-species plasma with ions of different charges. For $Z_{i} \gg 1$ e-i collisions dominate over e-e collisions and $k_{0, e} \propto \frac{1}{Z_{i}}$. For arbitrary $Z_{\text {eff }}$ the best approach would probably be to use $Z_{e f f}=\frac{\sum n_{i} Z_{i}^{2}}{n_{e}}$ instead of $Z_{i}$ in Eqs. (20) and (21). 


\section{References}

[1] Eich T et al 2011 Phys. Rev. Lett. 107, 215001

[2] Goldston R J 2012 Nuclear Fusion 52013009

[3] Spitzer L and Härm R 1953 Phys. Rev. 89977

[4] Braginskii S I 1965 Rev. Plasma Phys. 1205

[5] Stangeby P C 2000 The Plasma Boundary of Magnetic Fusion Devices, Institute of Physics Publishing, Bristol, UK

[6] Erents S K, Stangeby P C, LaBombard B et al 2000 Nuclear Fusion Vol. 40309

[7] Sun H J et al 2015 Plasma Phys. Control. Fusion 57125011

[8] Epperlein E M and Haines M G 1986 Phys. Fluids 291029

\section{Figures}

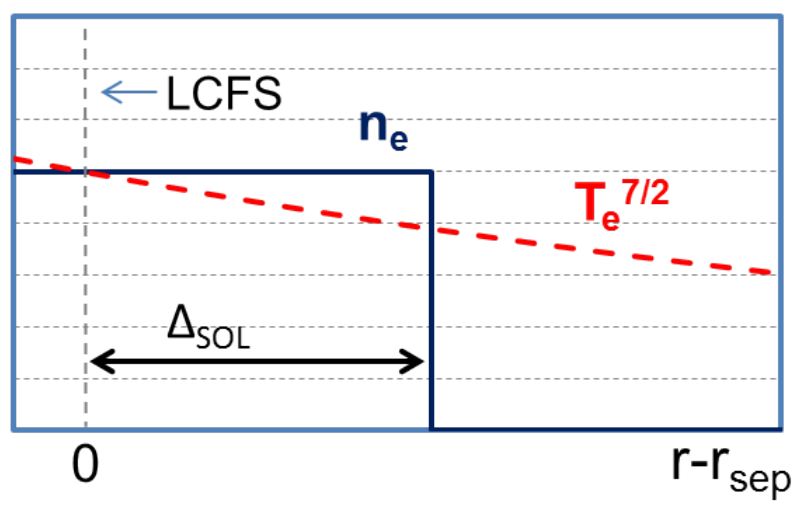

Figure 1. Idealistic SOL width, $\Delta_{\mathrm{SOL}}$, case

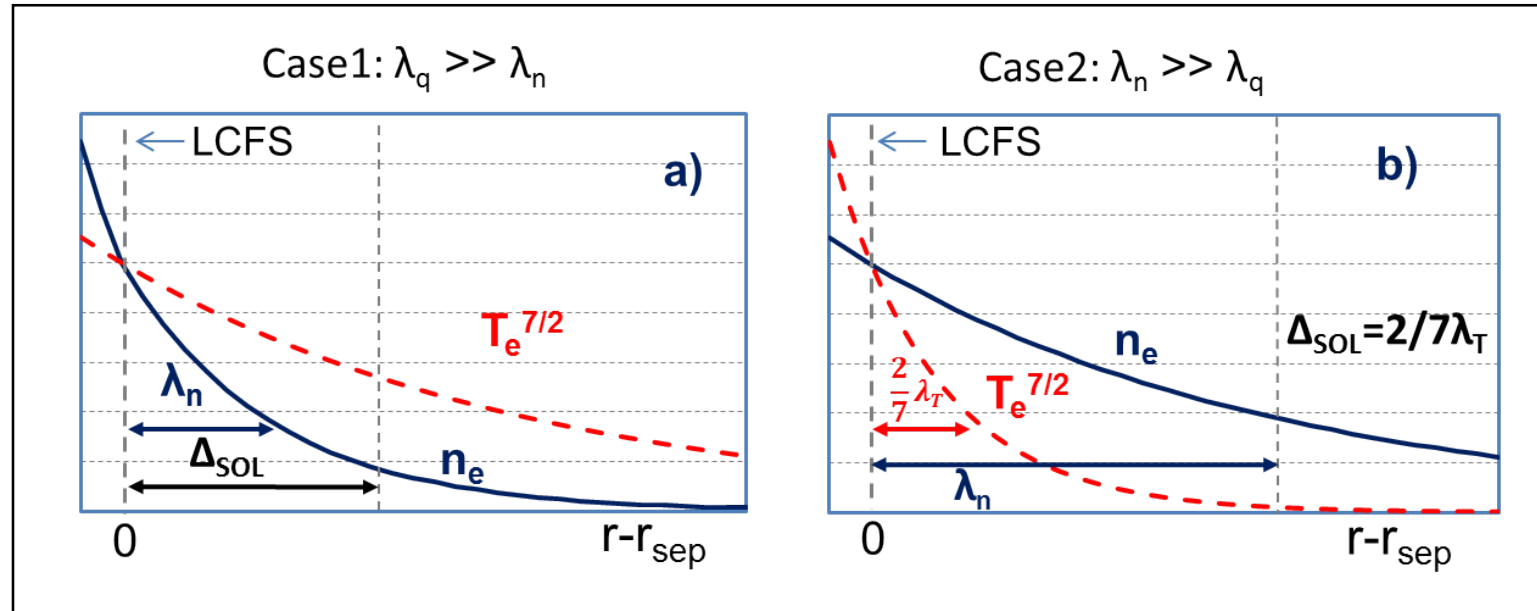

Figure 2: Two cases of the SOL profiles: fig2a) $\lambda_{q}>>\lambda_{n}$ and fig2b) $\lambda_{n}>>\lambda_{q}$ 